\title{
Clinical and genetic features of infancy- onset congenital myopathies from a Chinese paediatric centre
}

Yu Zhang ${ }^{1,2+}$, Hui Yan ${ }^{1 \dagger}$, Jieyu Liu ${ }^{1}$, Huifang Yan ${ }^{1}$, Yinan Ma ${ }^{3}$, Cuijie Wei ${ }^{1}$, Zhaoxia Wang ${ }^{4}$, Hui Xiong ${ }^{1}$ and Xingzhi Chang ${ }^{1 *}$

\begin{abstract}
Background: Congenital myopathies are a group of rare neuromuscular diseases characterized by specific histopathological features. The relationship between the pathologies and the genetic causes is complex, and the prevalence of myopathy-causing genes varies among patients from different ethnic groups. The aim of the present study was to characterize congenital myopathies with infancy onset among patients registered at our institution.
\end{abstract}

Method: This retrospective study enrolled 56 patients based on the pathological and/or genetic diagnosis. Clinical, histopathological and genetic features of the patients were analysed with long-term follow-up.

Results: Twenty-six out of 43 patients who received next-generation sequencing had genetic confirmation, and RYR1 variations (12/26) were the most prevalent. Eighteen novel variations were identified in 6 disease-causing genes, including RYR1, NEB, TTN, TNNT1, DNM2 and ACTA1. Nemaline myopathy (17/55) was the most common histopathology. The onset ages ranged from birth to 1 year. Thirty-one patients were followed for $3.83 \pm 3.05$ years (ranging from 3 months to 11 years). No patient died before 1 year. Two patients died at 5 years and 8 years respectively. The motor abilities were stable or improved in 23 patients and deteriorated in 6 patients. Ten (10/31) patients developed respiratory involvement, and 9 patients (9/31) had mildly abnormal electrocardiograms and/or echocardiograms.

Conclusion: The severity of congenital myopathies in the neonatal/infantile period may vary in patients from different ethnic groups. More concern should be given to cardiac monitoring in patients with congenital myopathies even in those with static courses.

Keywords: Congenital myopathy, Genetics, Infancy onset, Follow-up, Muscle biopsy

\section{Introduction}

Congenital myopathies are a group of rare genetic muscle disorders characterized clinically by generalized hypotonia and weakness, which generally occur from birth, and a static or slowly progressive course [1-3]. The classification of congenital myopathies is based mainly on the

\footnotetext{
*Correspondence: changxingzhi7318@163.com

†Yu Zhang and Hui Yan contributed equally to this work.

${ }^{1}$ Department of Paediatrics, Peking University First Hospital, No.1

Xianmen Street, Xicheng District, 100034 Beijing, PR China

Full list of author information is available at the end of the article
}

features observed in muscle biopsies. Accordingly, congenital myopathies are divided into the following forms: nemaline myopathy (NM), core myopathy, centronuclear myopathy (CNM), congenital fibre type disproportion (CFTD), and myosin storage myopathy [2]. To date, variations in at least 27 genes have been reported to cause congenital myopathy. Due to the use of next-generation sequencing, the number of variations related to congenital myopathy is expected to increase [4]. However, the relationship between the pathologies and the genetics of congenital myopathies is complex. Multiple genes 
can cause the same pathology, and variations in the same gene may cause different histopathologies. For example, CNM has been associated with at least seven genes, including MTM1, DNM2, RYR1, TTN, BIN1, CCDC78 and SPEG [4], whereas $R Y R 1$ variations can cause central core disease $(\mathrm{CCD})[5,6]$, multiminicore disease $(\mathrm{MmD})$ [7], core-rod myopathy [8], CNM [9] and CFTD [7]. In addition, patients with different pathological changes may present with similar clinical manifestations.

A static or slowly progressive course is recognized as a key clinical feature of congenital myopathies. Most patients with congenital myopathies will survive into adulthood, except when serious respiratory insufficiency and severe weakness are present in childhood $[2,3]$. Mortalities from congenital myopathies vary in different cohorts of patients [10-12]. For many neuromuscular diseases, cardiac involvement represents a major cause of morbidity and mortality [13]. However, the major cause of death in patients with congenital myopathies is respiratory failure. Except for a few cases related to TTN [14], $M H Y 7$ [15, 16] and FLNC [17] variations, patients with congenital myopathies usually have no or only mild cardiac affliction [18].

Using next-generation sequencing (NGS) techniques, many new genes are being identified as the causal genes of congenital myopathies as well as new variations identified in known genes, broadening the genotype spectrum of congenital myopathies [3]. The prevalence of myopathy-causing genes varies among patients from different ethnic groups. For example, variations in KLHL40 are a frequent cause of severe NM in Japanese patients [19], while TNNT1 variations are common among Amish people with severe NM [20]. Based on current knowledge of congenital myopathies, we retrospectively investigated 56 cases of infancy-onset congenital myopathies, including the clinical, pathological and genetic characteristics as well as long-term follow-up data. The present study emphasized infantile mortality and functional outcomes, combined with gene diversity.

\section{Patients and methods}

\section{Patients}

Fifty-six patients with congenital myopathy $(\mathrm{CM})$ who were registered from January 2007 to December 2019 (inclusive) in the Department of Paediatrics, Peking University First Hospital were included in this study. Patients with an infancy onset were defined as those exhibiting motor developmental delays in no more than 1 year of age. The clinical manifestation of the enrolled patients was consistent with the general presentation of congenital myopathies as follows: generalized muscle weakness and hypotonia; normal or mildly elevated serum creatine kinase (CK); and normal or mild myopathic changes on electromyogram examination. In addition, the patients had either characteristic histopathological features of congenital myopathies, definitive genetic diagnosis or both. One case (Pt 3) was previously reported in our case report in 2013, and clinical manifestation and family segregation were presented without follow-up [21]. NM patients (Pt 10-11 and Pt 35-38) were included in one of our previous studies focused on the genetic features of NM patients with onset ages ranging from infancy to adulthood [22]. Open muscle biopsies were performed in 55 patients ( $98.2 \%$, one patient refused), and pathological diagnosis was made according to the criteria suggested by Dubowitz et al. [23]. Genetic analysis was performed in 43 patients (some patients diagnosed at earlier times when commercial NGS was not available were lost to follow-up), and 31 patients were followed up. Written informed consent was obtained from all patients and/or their parents prior to inclusion in the study. The present study was approved by the Ethics Committee of Peking University First Hospital (Beijing, China, Approved number: 2018-265).

\section{Muscle biopsy}

Open muscle biopsies were taken from the biceps or quadriceps femoris. Fresh frozen muscle specimens were fixed in liquid nitrogen, and frozen sections $(8-10 \mu \mathrm{m})$ were processed. A series of histochemical methods was used in all muscle specimens, including haematoxylin and eosin (H\&E), modified Gomori trichrome (MGT), oil Red O, periodic acid-Shiff (PAS), nicotinamide adenine dinucleotide dehydrogenase-tetrazolium reductase (NADH-TR), succinate dehydrogenase (SDH), cytochrome c oxidase (COX), nonspecific esterase and adenosine triphosphatase (ATPase) staining. The ultrastructure was observed under transmission electron microscopy (JEM1230, Japan). The pathological categories were classified according to the criteria suggested by Dubowitz et al. [23]: (1) core myopathies including CCD and MmD; (2) NM; (3) CNM; (4) CFTD; and (5) nonspecific myopathic changes (NSMC). We included patients with mixed structural pathological features, for example, a combination of cores and rods. However, we did not consider type 1 fibre dominance/uniformity as an isolated diagnosis due to its low specificity and potential coexistence with other more specific features.

\section{Gene variation analyses}

Blood samples were collected, and genomic DNA was extracted from leukocytes of patients. The coding exons of the 169 myopathy-causing genes (Supplement 1) were selected by a gene capture strategy (Kangso Medical Inspection, China; MyGenostics, Beijing). The gene panel was used to screen for variations causing hereditary 
myopathies, and high coverage $(\geq 100 \times)$ sequencing was used to detect single nucleotide polymorphisms (SNPs) and indel variations. The variations taken into further consideration were as follows: (1) marked as a pathogenic variation in the Human Genome Mutation Database (HGMD); (2) nonsense variations; (3) frameshift variations; and (4) located in an essential splice site. The pathogenicity of nonsynonymous variation was evaluated by using multiple algorithms, including SIFT, Ployphen-2, Mutation-Taster, PROVEAN and Splice-Site Prediction by Neural Network. When pathogenic variations were not found in some patients through panel screening, whole exome sequencing was used for further study. The exomes were captured using the xGen Exome Research Panel v1.0 (Integrated DNA Technologies) and sequenced on an Illumina HiSeq2000 (Illumina, San Diego, CA, USA) with 100-bp paired-end reads (Kangso Medical Inspection, China; MyGenostics, Beijing). After the sequencing, the raw data were saved as a FASTQ format, and then we did the bioinformatics analysis. First, Illumina sequencing adapters and low-quality reads $(<80 \mathrm{bp})$ were filtered by cut adapt. After quality control, the clean reads were mapped to the UCSC hg19 human reference genome using BWA. Duplicated reads were removed using picard tools, and mapping reads were used for variation detection. Second, the variations of SNP and InDel were detected by GATK Haplotype Caller, then we used GATK Variant Filtration to filter variations. The filtered standards were as follows: a) variations with mapping qualities <30; b) the Total Mapping Quality Zero Reads $<4$; c) approximate read depth $<5$; d) $\mathrm{QUAL}<50.0$; e) Phred-scaled $p$-value using Fisher's exact test to detect strand bias $>10.0$. After above two steps, the data would be transformed to VCF format. Variations were further annotated by ANNOVAR and associated with multiple databases, such as 1000 genome, ESP6500, dbSNP, EXAC, Inhouse (MyGenostics), HGMD, as well as predicted by SIFT, PolyPhen-2, MutationTaster, GERP++ . Then four steps were used to select the potential pathogenic variations in downstream analysis: (i) variation reads should be more than 5 , variation ration should be no less than $30 \%$; (ii) removing the variations with frequency of more than 5\% in 1000 genome, ESP6500 and Inhouse database; (iii) If the variations existed in InNormal database (MyGenostics), then dropped; (iv) the synonymous variations reported in HGMD were left, and the remaining of the synonymous variations were excluded. All candidate pathogenic variations were confirmed by Sanger sequencing. The interpretation of the variations was made according to the guidelines provided by the American College of Medical Genetics (ACMG) [24].

\section{Patient follow-up}

Patients were followed up at our outpatient department. Mobility and musculoskeletal complications were evaluated with physical examinations. The manual muscle test (MMT) was used to assess the degree of muscle weakness. Posteroanterior radiographs were performed in selected patients with suspected scoliosis, and a Cobb angle of more than $10^{\circ}$ was considered significant. Mild scoliosis was defined as a Cobb angle ranging from $10^{\circ}$ to $40^{\circ}$, and severe scoliosis was defined as a Cobb angle over $40^{\circ}$. Forced vital capacity (FVC) was measured and expressed as a percentage of the predicted value for height in patients over 6 years old. Cardiac function was monitored with electrocardiograms (ECGs) and echocardiograms.

\section{Results}

\section{Gene variations}

Pathogenic variations were identified in 26 out of the 43 patients $(26 / 43,60.5 \%)$. In total, 40 pathogenic and likely pathogenic variants were confirmed in 6 myopathy-related genes (Table 1 and Supplement 2). Of them, 18 variants were novel, including eight $R Y R 1$ variations (c.3880G > T, c.658C > T, c. $4715 \mathrm{~T}>\mathrm{C}, \mathrm{c} .4454 \mathrm{G}>\mathrm{A}$, c. $2044 \mathrm{C}>\mathrm{G}$, c.6823G $>$ A, c. 1675 dup and c.7330C $>$ T), two $N E B$ variations (c.3567+1G>A and c.6734dup), one $A C T A 1$ variation (c.402G $>\mathrm{T}$ ), one $D N M 2$ variation (c.1893+1G > A), four TTN variations (c.32312-1G >A, c.2099_2106dup, c.85818 T > A and c.102798-102800del), and two TNNT1 variations (c.1A > G and c.353delC). Variations in RYR1 were identified in 12 patients $(12 / 43$, $27.8 \%$ ), being the most frequent gene variations in both dominant and recessive inheritance. All dominant $R Y R 1$ variations were pathologically associated with $C C D$, and recessive variations were associated with different pathological changes (MmD, 3 cases; CFTD, 1 case; and CNM 1 case). Variations associated with NM were identified in three genes, including compound heterozygous $N E B$ variations in 4 patients $(4 / 43,9.3 \%)$, heterozygous ACTA1 variations in 4 patients $(4 / 43,9.3 \%)$ and compound heterozygous TNNT1 variations in one patient (1/43, 2.3\%). Compound heterozygous TTN variations were found in three patients $(3 / 43,7 \%)$ with different pathological diagnoses (MmD, 2 cases; and CNM, 1 case). Heterozygous $D N M 2$ variations were found in 2 patients $(2 / 43,4.7 \%)$ with CNM.

\section{Pathologic characteristics}

A total of 55 muscle specimens were collected. $\mathrm{NM}(30.9 .8 \%, 17 / 55)$ was the most frequent 


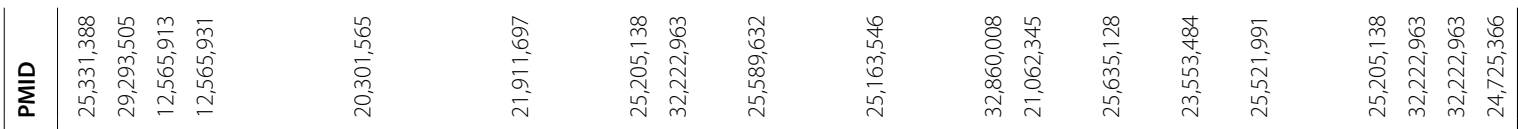

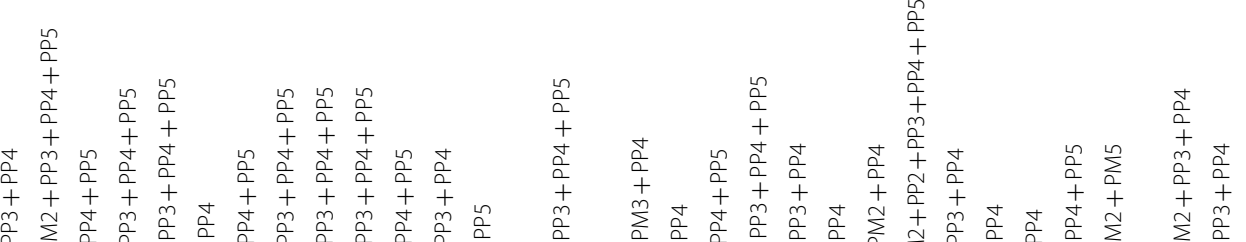

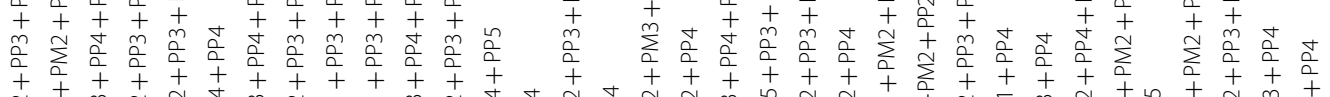

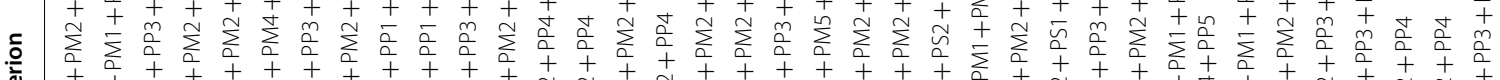

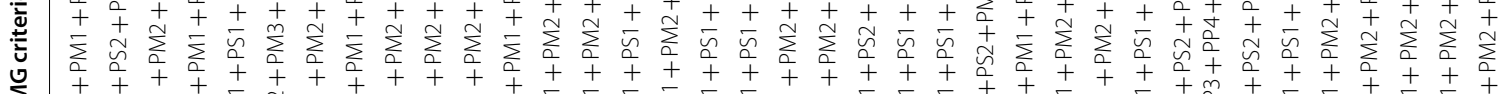

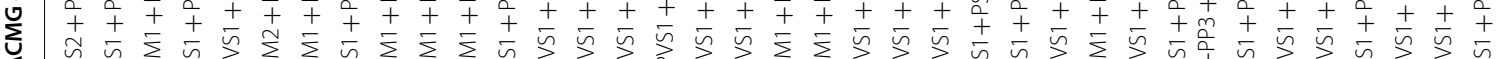

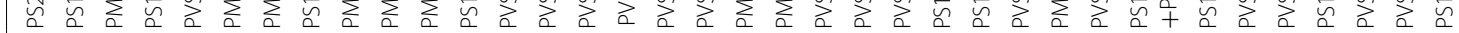

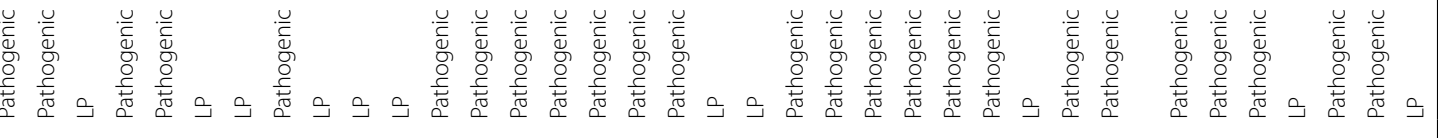

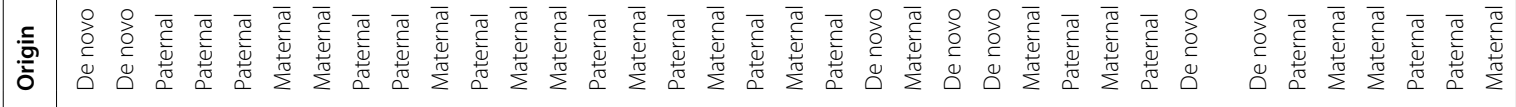

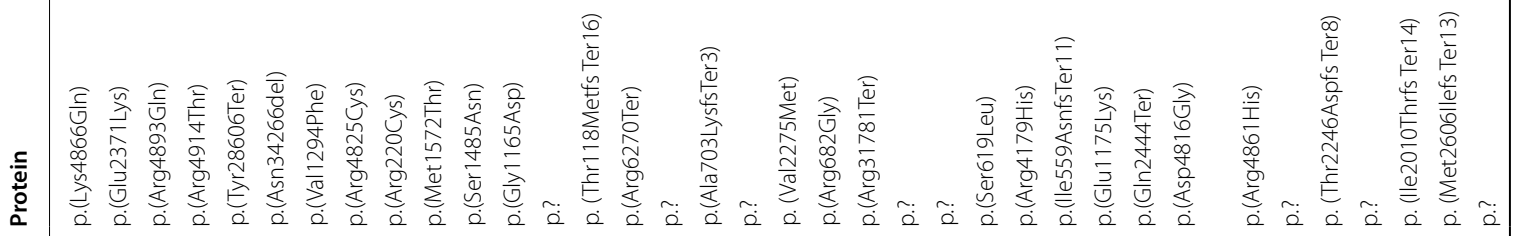

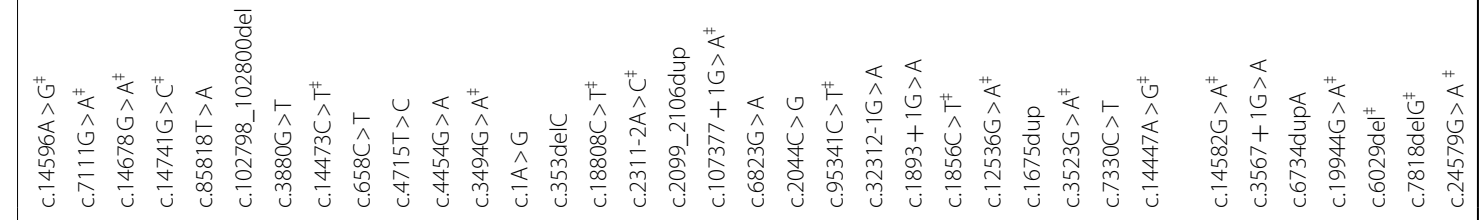

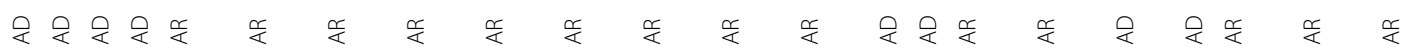

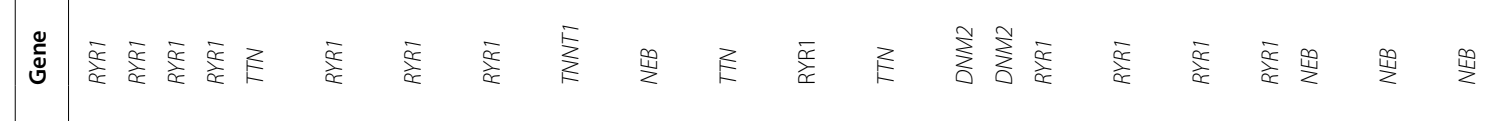

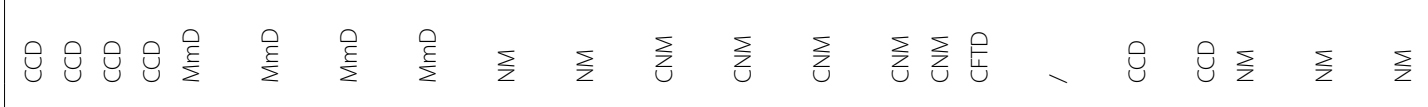




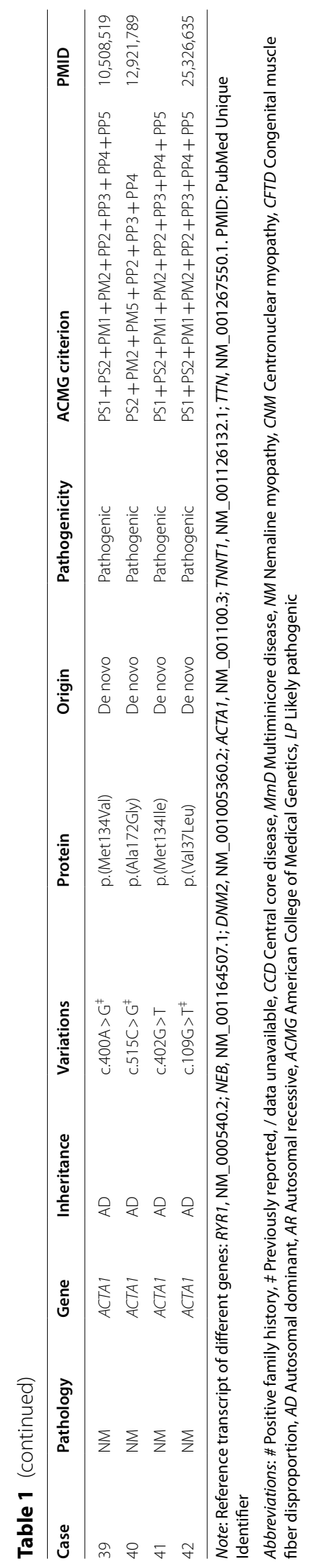


histopathological diagnosis in this cohort followed by core myopathy $(29.1 \%, 16 / 55)$. Typical nemaline rods (Fig. 1) were the main pathological findings in 17 patients. Central cores (Fig. 2A, C) were observed in 10 patients, and multiple minicores (Fig. 2B, D) were noted in 6 patients. The coexistence of cores and rods was observed in only one patient (Pt 19). Characteristic changes of CNM were found in 10 patients $(18.2 \%$, $10 / 55)$, including central nuclei in a large number of muscle fibres $(>30 \%)$, associated with radial arrangements of sarcoplasmic strands in patients with DNM2 mutations (Fig. 3A, B). CFTD was diagnosed in 11 patients $(20 \%, 11 / 55)$. The key pathological change was the presence of type 1 fibres that were at least $12 \%$ smaller than type 2 fibres without other significant histological abnormalities (Fig. 3C).

\section{Clinical features}

The enrolled 56 patients with congenital myopathies included 32 boys and 24 girls (Table 2), and they were diagnosed as follows: 25 patients were diagnosed by characteristic pathological features and pathogenic gene variations; 30 patients were diagnosed by typical histopathologic features; and one patient was genetically diagnosed without muscle pathology. We followed up with $31(31 / 56,55.4 \%)$ patients for $3.83 \pm 3.05$ years (ranging from 3 months to 11 years) (Table 2). Seven patients had a significant family history, which suggested that the inheritance pattern was autosomal dominant in 3 patients and autosomal recessive in 4 patients. Premature death in family members was noted in 4 families. There was no history of parental consanguinity and no history of miscarriage or abortion in the family.

Thirty-four patients $(34 / 56,60.7 \%)$ who had an earlier onset age of less than 6 months presented with pronounced generalized hypotonia, weakness and motor milestone delay. Eight patients $(14.3 \%, 8 / 56)$ had bulbar involvement manifested with dysphagia and congenital laryngeal stridor, and of these patients, four had sucking failure requiring nasogastric tube feeding for up to 8 months. Two patients had respiratory insufficiency requiring short-term ventilator support (Pts 53 and 54). Twenty-two $(39.3 \%, 22 / 56)$ patients who had an onset age over 6 months presented with climbing difficulties, abnormal gait or a tendency to fall frequently compared to their peers. In total, three patients presented with congenital hip dysplasia, and one patient had lordosis and knee contracture at the first evaluation. Reduced foetal movements were noted in three patients, and premature birth was noted in the other three patients.

The diagnostic ages ranged from 5 months to 16 years $(5.53 \pm 3.66 \mathrm{y})$. Although 34 patients had pronounced weakness before the age of 6 months, only 4 patients (Pts 7, 53, 54 and 55) were diagnosed before the age of 13 months. All 4 patients had severe weakness with bulbar and respiratory involvement at birth. One patient (Pt 7) had a positive family history as the elder sister of Pt 7 died shortly after birth due to pulmonary failure and severe muscle weakness.

Generalized muscle weakness, as an initial symptom, was noted in the majority of patients $(55 / 56,98.2 \%)$, and it was more severe in the lower limbs and more prominent in the proximal muscles. One patient (Pt 15) with centronuclear myopathy due to DNM2 heterozygous variation presented with predominant weakness of the distal lower limbs. Three patients showed prominent axial muscle weakness from the early onset of the disease. Twenty-seven $(27 / 56,48.2 \%)$ patients showed facial muscle weakness, manifesting as an elongated face, high-arched palate, tented upper lip, micrognathia, nasal tone vocalization and slurred speech. Disorders of ocular motility with eyelid ptosis were found
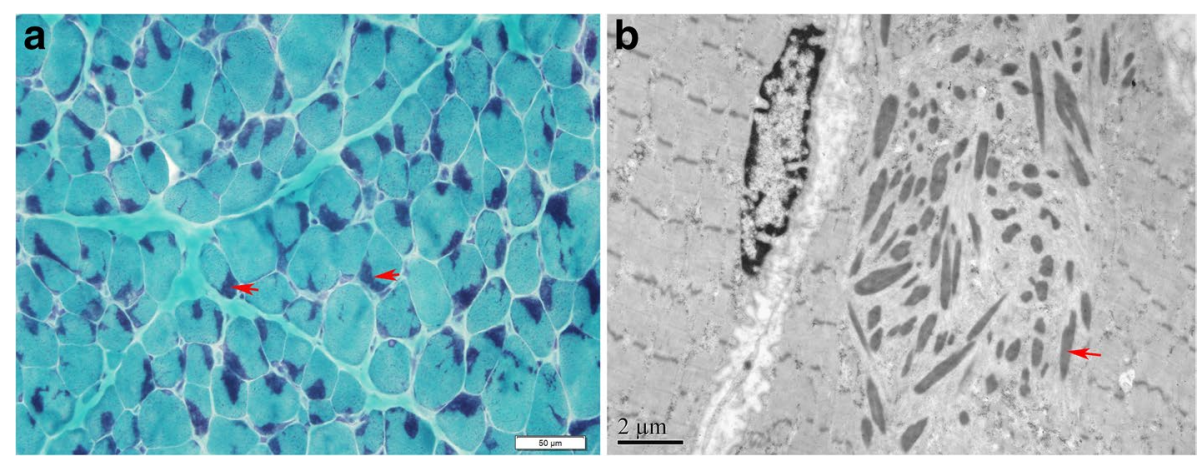

Fig. 1 Nemaline rods in NM patients (A, patient 46, right quadriceps femoris). Transverse sections of muscle specimens stained with modified Gomori trichrome show numerous nemaline rods (arrows) clustered under the sarcolemmal membrane in muscle fibres. (B, patient 46, right quadriceps femoris) Electron microscopy shows many typical rods with disorganized myofibrils, and the rods have the same electron density as the Z-disk (arrow) 

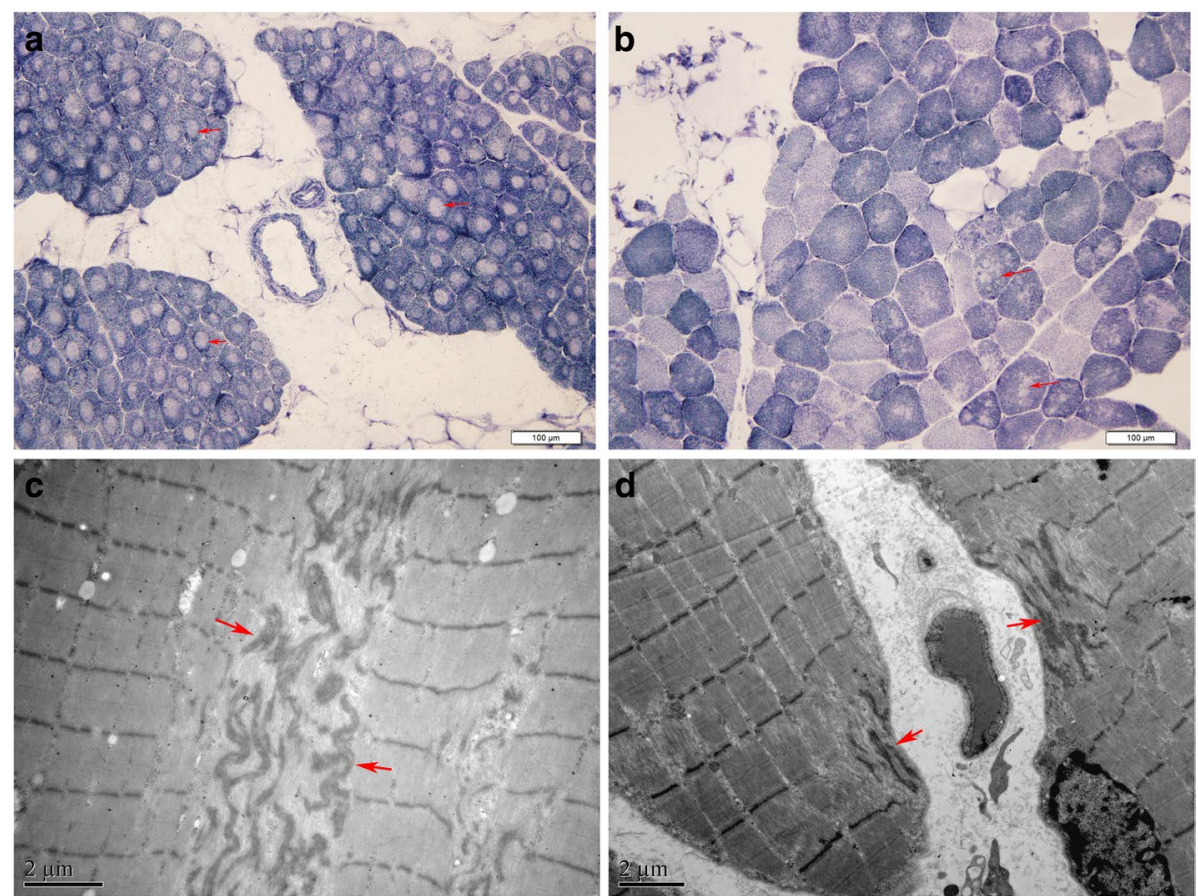

Fig. 2 Central cores and minicores in patients with core myopathies. (A, patient 1, left biceps) Reduced nicotinamide adenine dinucleotidetetrazolium reductase (NADH-TR) staining of transverse sections of muscle specimens shows the typical central cores. The cores are single, central placed, well-circumscribed, circular regions (arrows) that appeared in almost all fibres. (B, patient 6, right quadriceps femoris) Minicores are demonstrated as multiple foci of ill-defined small areas with deficiency of oxidative activity on NADH-TR staining (arrows). (C, patient 1, left biceps) Electron microscopy shows that the central cores (arrows) contain disorganized myofibrils with Z-line streaming. (D, patient 6, right quadriceps femoris) Electron microscopy shows two minicores (arrows) at the periphery of muscle fibres

in 2 patients (Pts 2 and 54). With the development of the disease, additional involvement of axial and distal muscles was common in patients with follow-ups. Seventeen patients $(17 / 31,54.4 \%)$ developed variable scoliosis, and one patient (Pt 14) showed spinal rigidity. Six patients $(6 / 31,19.4 \%)$ demonstrated multiple joint contractures, and 17 patients $(17 / 31,54.8 \%)$ showed tightness of the Archilles tendon. Three patients had hypertrophy of the gastrocnemius.

Muscle weakness improved gradually in all patients during the neonatal and infancy periods. Eight patients $(8 / 56)$ had bulbar involvement at onset, and four of them required nasogastric tube feeding. Bulbar impairment improved gradually, and no patient required tube feeding
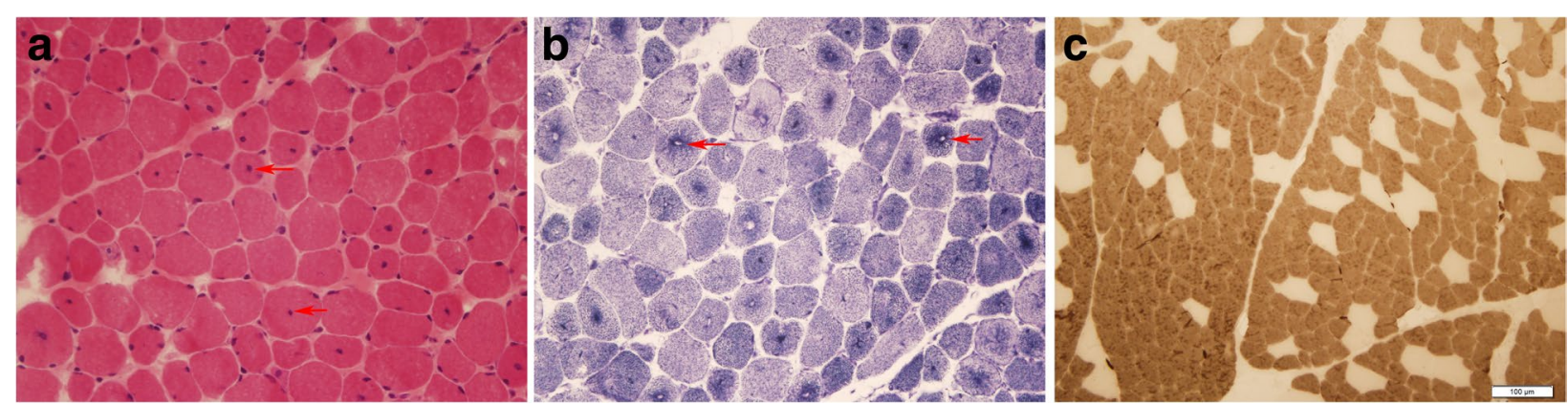

Fig. 3 Transverse sections of muscle specimens from patients with CNM and CFTD. (A, patient 15, left biceps) Central nuclei (arrows) appeared in more than $30 \%$ of fibres (haematoxylin and eosin staining). (B patient 15, left biceps) The intermyofibrillar network revealed by reduced nicotinamide adenine dinucleotide-tetrazolium reductase (NADH-TR) staining, showing radiates like spokes of a wheel from the centre to the periphery of the fibres (arrows). (C, patient 17, left biceps) Adenosine triphosphatase (ATPase) staining with preincubation at pH4.6. Type 1 fibers (dark type) are at least $12 \%$ smaller than type 2 fibres (pale type) accompanied by type 1 fibre predominance 
after 8 months of age. No gastrostomy was performed. Three patients were lost to follow-up before they gained walking ability (at 10-13 months). Forty-three (43/56, $76.8 \%)$ patients achieved independent ambulation before 2 years of age, and 7 patients achieved independent ambulation at 2.5-5 years. Moreover, 3 patients (Pts 2, 24 and 42) never achieved independent ambulation by their last visit at 3-3.3years. Among the patients with followups, 23 patients $(74.2 \%, 23 / 31)$ remained ambulatory, but some of them had an unstable gait and experienced frequent falls. Six $(6 / 31,19.4 \%)$ patients gradually lost ambulation at an average age of $11.02 \pm 3.05$ years (ranging from 8 to 17 years), who initially acquired independent ambulation. Severity variance was noted between $R Y R 1$ patients with dominant and recessive inheritance. Of the four patients with dominant $R Y R 1$ variations, one (Pt 2) could only sit with support at her last visit (3years old), one lost ambulation at 12 years, one lost ambulation at 17 years, and one could walk independently at 18 years. All six patients with recessive variations walked independently, and 5 of them were more than 13 years of age (ranging 13 to 18 years) (Table 2).

Two patients (Pts 53 and 54) with CFTD developed respiratory insufficiency, requiring continuous positive airway pressure therapy immediately after birth, but they gradually improved to discontinue the need for ventilators. No patients died within their first year of life. During follow-ups, respiratory problems were found in ten $(32.3 \%, 10 / 31)$ patients after infancy. Two out of the patients (Pts 19 and 20) required night intermittent ventilator support (one at 8 years old and one at 9 years old). Two patients (Pts 26 and 28) died of respiratory failure at 8 and 5 years old, respectively. Five patients had decreased forced vital capacity (FVC) to $30-72.5 \%$ of expected values. One patient (Pt 27) complained of mild breathing difficulty without an FVC measurement. NM (4/10) was more frequently related to respiratory insufficiency later, and CFTD was associated with early-onset respiratory failure as the two patients with respiratory insufficiency at birth had the same CFTD.

No child complained of cardiac symptoms. Fifty-five $(55 / 56,98.3 \%)$ patients had normal echocardiograms with or without electrocardiogram at initial evaluation, except for one patient (Pt 45) who visited our hospital for the first time at 9 years old with respiratory insufficiency and mild pulmonary hypertension. Thirtyone patients received repeated echocardiogram and electrocardiogram examinations during follow-ups. Seven patients had mildly abnormal electrocardiogram results, including sinus tachycardia (in 3 cases), $\mathrm{PR}$ prolongation (in 1 case), QT interval prolongation (in 3 cases) and changes in ST segment and $\mathrm{T}$ waves (in
2 cases) (some patients had multiple abnormal electrocardiogram changes). Two patients had mild interventricular septal hypertrophy. In total, 9 patients (29\%, 9/31) showed mild abnormal electrocardiograms and/ or echocardiograms. $\mathrm{MmD}(4 / 9,44.4 \%)$ was found to be the most frequent pathological diagnosis in patients with mild cardiac abnormalities. Gene variations in RYR1 ( 2 cases), TTN ( 2 cases) and TNNT1 (1 Case) were identified in 5 of these patients.

During the follow-up, three patients (3/31) had slurred speech and mild difficulty swallowing large chunks. Three patients were underweight (below the 3rd centile), and 2 were overweight (above the 97th centile). Twenty-three patients (23/31) were studying at normal public school, and no one complained of learning difficulty. Eight patients were not in school due to the following reasons: five of the patients were under school age, and the remaining three did not go to school due to walking difficulties. No malignant hyperthermia was noted in the patients with $R Y R 1$ variations.

\section{Discussion}

Congenital myopathies are a rare group of muscle diseases with clinical and genetic heterogeneity [3]. In this study, we analyzed the clinical, genetic and pathological data of patients with infancy-onset congenital myopathies. The most common presentations in our patients were generalized muscle weakness and hypotonia as well as proximally predominant limb weakness combined with axial muscle involvement, which was consistent with other studies $[10,11]$. However, the frequency of neonatal bulbar involvement and respiratory failure (13.8\%) was significantly lower than that in other studies, which may contribute to the low infant mortality in this cohort. Colombo et al. reported that $30.4 \%(38 / 125)$ of patients required respiratory support at birth, and that $25.2 \%$ required nasogastric feeding at birth [10]. Another study has reported a mortality rate for congenital myopathies of $8 \%(5 / 66)$ with 4 out of 5 deaths occurring within the first 2 months of life [11]. Compared to these studies, none of our patients died during infancy. There are several reasons for this discrepancy. The discrepancy is mainly due to the underlying genes as there is a different prevalence of myopathy-causing genes among patients from different ethnic groups [19, 20]. It has been reported that severe neonatal bulbar and respiratory muscle involvement occurs particularly in severe NM (most likely ACTA1-, NEB- or KLHL40related) and MTM1-related myotubular myopathy [1, 12, $25,26]$. XMTM1 gene variations are recognized as the most lethal cause of congenital myopathy in infancy [12]. In Colombo's cohort, the variations in ACTA1, MTM1 or KLHL4O accounted for a large portion of the patients 


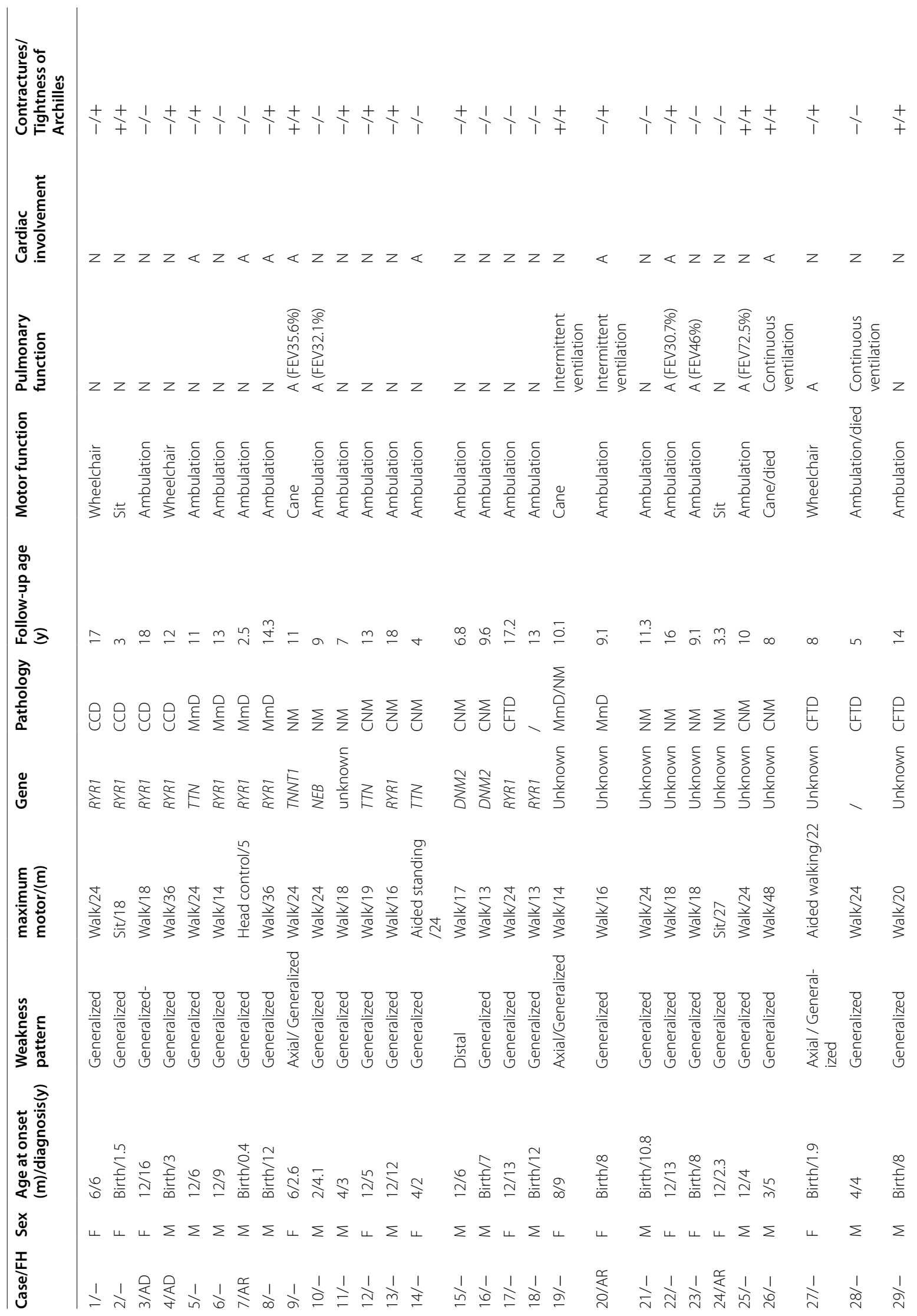




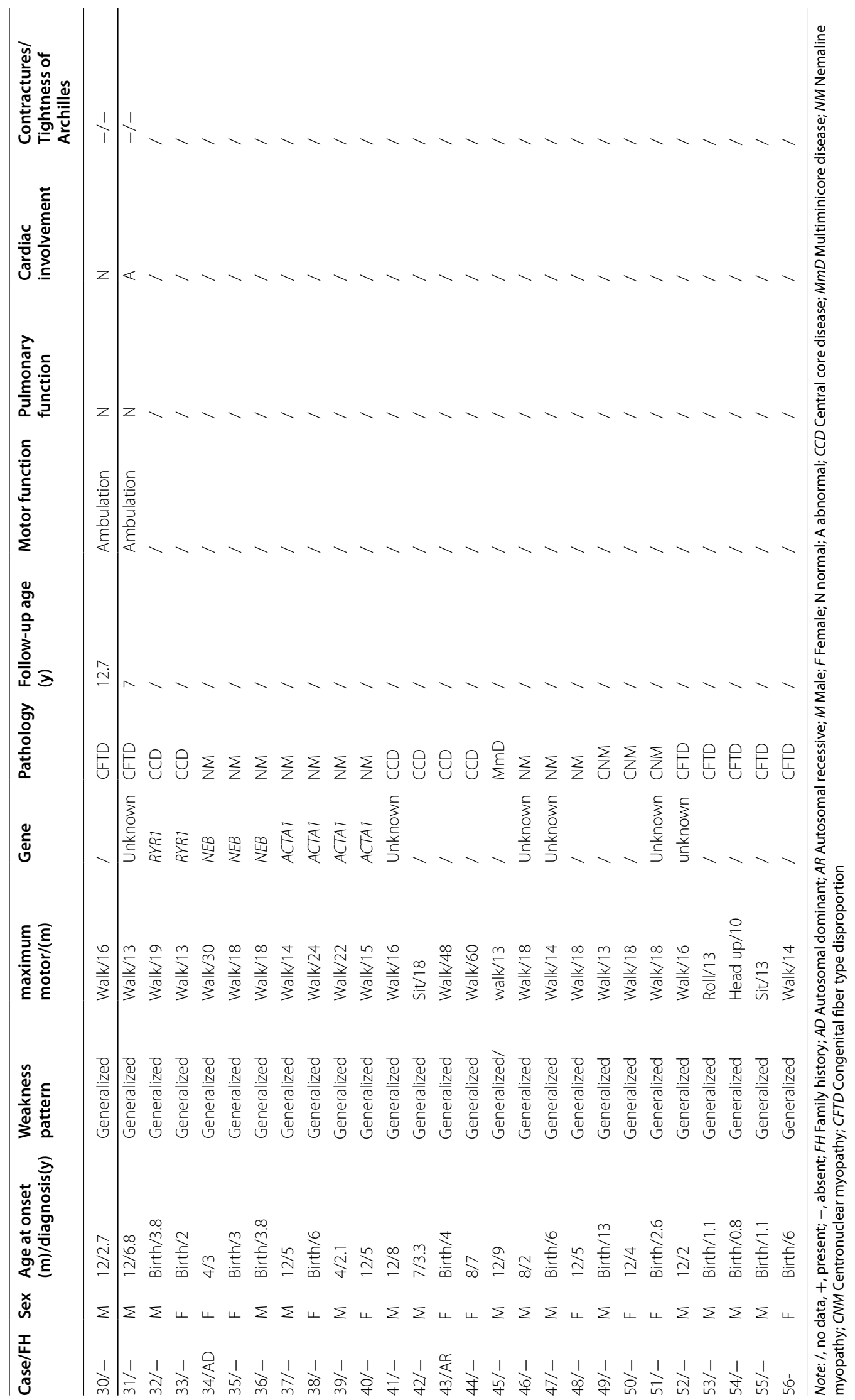


with severe bulbar involvement and caused a $12 \%$ death rate, mainly within the first year. Comparatively, only four ACTA1 pathogenic variations were identified in our patients, and MTM1 or KLHL4O pathogenic variations were not found. Moreover, sampling bias should be noted. The present study was performed at a single paediatric centre in China. Most of our patients came to us from all across mainland China, and the patients with lethal complications during the perinatal period might be absent from the present study. There may have been a few patients who died of severe congenital myopathies before visiting us.

In contrast to many other neuromuscular diseases, cardiac involvement represents a major cause of morbidity and mortality [13]. Cardiac involvement is not an important issue in most cases of congenital myopathies [3]. Congenital myopathies are generally associated with a mild cardiac phenotype [18], and lethal cases have rarely been reported [27, 28]. None of our patients complained of cardiac symptoms, and only mildly abnormal changes on electrocardiograms and/or echocardiograms were detected. The mildness of cardiac involvement in our patients was consistent with that in previous reports [18, 27]. TTN and $M Y H 7$ are the most frequent genes related to severe cardiac involvement in the literature [29], whereas ACTA1, RYR1, TPM2, FLNC and SPEG have occasionally been reported [15, 17, 27, 30]. Although 2 out of 3 TTN patients were found to have mild cardiac abnormalities in our cohort, it was difficult to analyze the severity of cardiac involvement between different gene variations due to the small sample size. Considering the relatively high frequency $(29 \%, 9 / 31)$ of mild cardiac involvement in our cohort, our observation suggested that cardiac involvement in congenital myopathies may be underestimated. The issue of cardiac involvement in congenital myopathies and its relationship with different gene variations needs further study.

Core myopathy and NM were the most frequent pathologies in our cohort, and RYR1 was the most common associated gene, similar to previous reports but with varied rates between studies [10,11]. Heterozygous dominant $R Y R 1$ variations were pathologically associated with $C C D$, and recessive $R Y R 1$ variations were associated with different pathological changes, including $\mathrm{MmD}, \mathrm{CNM}$ and CFTD, which was consistent with previous reports [7, 9, 31, 32]. Patients with $R Y R 1$ variations have a wide clinical spectrum with variable severity $[33,34]$. It has been reported that dominant $R Y R 1$ variations are associated with milder phenotypes, and patients with recessive RYR1 variations have earlier onset, more weakness and functional limitations [32]. It was difficult to fully discriminate the presentations of dominant and recessive RYR1 variations due to the small sample size in our study. However, different from previous reports, the motor ability of the patients with dominant $R Y R 1$ variations was worse than that of patients with recessive variations in our follow-up patients. We believe that the clinical heterogeneity of $R Y R 1$ requires more research.

Novel complex heterogeneous TNNT1 variations were identified in one of our patients (Pt 9). TNNT1 was first identified in Order Amish patients with NM as the causing gene [20]. The TNNT1 c.505G $>\mathrm{T}$ variation has a carrier frequency of $6.5 \%$ within Old Order Amish settlements of North America [35]. In their first months of life, afflicted Amish infants have tremors with hypotonia and mild contractures of the shoulders and hips followed by progressive muscle weakness, atrophy and contractures. Early respiratory failure and striking stiffness of the cervical spine usually cause death in the second year [20,35]. The prominent axial muscle involvement and multiple contractures in the hips, shoulders, elbows and knees in Pt 9 were similar to those of Amish patients. However, Pt 9 presented with hypotonia and motor delay from the age of 6 months with a normal neonatal period and without tremors throughout the clinical course. Pt 9 was still alive at 11 years old with supported walking, severe scoliosis and restrictive respiratory insufficiency. The symptoms of Pt 9 were much milder than that of Amish patients. In addition, most of the reported TNNT1 variations were homozygous null variations [36], whereas one paternal frameshift variation (c.353delC; p. Thr118MetfsTer16) and one maternal missense variation (c.1A > G; p. Met1Val) were identified in our patients, which expanded the genotype of TNNT1.

\section{Conclusion}

The present study expanded the clinical and genetic spectrum of congenital myopathies. First, we reported 18 novel variations in 6 myopathy-causing genes, including RYR1, NEB, ACTA1, DNM2, TTN and TNNT1, and we described the clinical discrepancy between our patients and previous reports, especially in RYR1 - and TNNT1 - related myopathy. Second, in addition to routine monitoring of muscular complications, ambulatory loss and respiratory failure, our follow-up data provided more details of nonmuscle involvements in patients with congenital myopathies. The necessity of cardiac function evaluation is emphasized, even in patients with static courses. 


\section{Abbreviations}

AD: Autosomal dominant; AR: Autosomal recessive; ATPase: Adenosine triphosphatase; CCD: Central core disease; CFTD: Congenital fibre type disproportion; CNM: Centronuclear myopathy; COX: Cytochrome c oxidase; FVC: Forced vital capacity; H\&E: Haematoxylin and eosin; MGT: Modified Gomori trichrome; MmD: Multiminicore disease; NADH-TR: Nicotinamide adenine dinucleotide dehydrogenase-tetrazolium reductase; NM: Nemaline myopathy; PAS: Periodic acid-Shiff; SDH: Succinate dehydrogenase; WES: Whole exome sequencing.

\section{Supplementary Information}

The online version contains supplementary material available at https://doi. org/10.1186/s12887-021-03024-0.

\section{Additional file 1.}

\section{Additional file 2.}

\section{Acknowledgements}

We thank all the patients and their families. We thank Wei Wei in Kangso Medical Inspection, and Junying Liu, Ran Liu in MyGenostics, Beijing, China, for their technical support with the gene variation analysis.

\section{Authors' contributions}

CX had full access to all of the data and take responsibility for the accuracy of the data analysis. Acquisition, analysis and interpretation of clinical and pathologic data: ZY, $L$ J and WC. Analysis of genetic data: YH, MY and WZ. Drafted the paper: $\mathrm{CX}$ and $\mathrm{YH}$. Provided comments and revised the paper: $\mathrm{YH}, \mathrm{CX}$ and $\mathrm{XH}$. The author(s) read and approved the final manuscript.

\section{Funding}

Not applicable.

\section{Availability of data and materials}

The datasets used and/or analyzed during the present study are available from the corresponding author on reasonable request. Novel gene variations have been submitted to the ClinVar database (https:// www.ncbi.n/m.nih.gov/clinvar/).

\section{Declarations}

\section{Ethics approval and consent to participate}

This study was approved by the Ethics Committee of Peking University First Hospital (Beijing, China, approval number 2018-265). The patients and/or their parents provided their written informed consent to participate in this study. All methods were performed in accordance with the relevant guidelines and regulations.

\section{Consent for publication}

Not applicable.

\section{Competing interests}

The authors declare that they have no competing interests.

\section{Author details}

${ }^{1}$ Department of Paediatrics, Peking University First Hospital, No.1 Xianmen Street, Xicheng District, 100034 Beijing, PR China. ${ }^{2}$ Department of Paediatrics, Peking University International Hospital, 102206 Beijing, PR China. ${ }^{3}$ Department of Central Laboratory, Peking University First Hospital, 100034 Beijing, PR China. ${ }^{4}$ Department of Neurology, Peking University First Hospital, 100034 Beijing, PR China.

Received: 22 March 2021 Accepted: 18 November 2021

Published online: 26 January 2022

\section{References}

1. North $\mathrm{KN}$, Wang $\mathrm{CH}$, Clarke N, Jungbluth $\mathrm{H}$, Vainzof M, Dowling JJ, et al. Approach to the diagnosis of congenital myopathies. Neuromuscul Disord. 2014;24(2):97-116.
2. Cassandrini D, Trovato R, Rubegni A, Lenzi S, Fiorillo C, Baldacci J, et al. Congenital myopathies: clinical phenotypes and new diagnostic tools. Ital J Pediatr. 2017:43(1):101.

3. Claeys KG. Congenital myopathies: an update. Dev Med Child Neurol. 2020;62(3):297-302.

4. Pelin K, Wallgren-Pettersson C. Update on the genetics of congenital myopathies. Semin Pediatr Neurol. 2019;29:12-22.

5. Gu M, Zhang S, Hu J, Yuan Y, Wang Z, Da Y, et al. Novel RYR1 missense mutations in six Chinese patients with central core disease. Neurosci Lett. 2014;566:32-5

6. Wu S, Ibarra MC, Malicdan MC, Murayama K, Ichihara Y, Kikuchi H, et al. Central core disease is due to RYR1 mutations in more than $90 \%$ of patients. Brain. 2006;129(Pt 6):1470-80.

7. Lawal TA, Todd JJ, Meilleur KG. Ryanodine receptor 1-related myopathies: diagnostic and therapeutic approaches. Neurotherapeutics. 2018;15(4):885-99.

8. Hernandez-Lain A, Husson I, Monnier N, Farnoux C, Brochier G, Lacene E, et al. De novo RYR1 heterozygous mutation (14898T) causing lethal corerod myopathy in twins. Eur J Med Genet. 2011;54(1):29-33.

9. Abath Neto O, Moreno CAM, Malfatti E, Donkervoort S, Bohm J, Guimaraes JB, et al. Common and variable clinical, histological, and imaging findings of recessive RYR1-related centronuclear myopathy patients. Neuromuscul Disord. 2017;27(11):975-85.

10. Colombo I, Scoto M, Manzur AY, Robb SA, Maggi L, Gowda V, et al. Congenital myopathies: natural history of a large pediatric cohort. Neurology. 2015;84(1):28-35

11. Maggi L, Scoto M, Cirak S, Robb SA, Klein A, Lillis S, et al. Congenital myopathies--clinical features and frequency of individual subtypes diagnosed over a 5-year period in the United Kingdom. Neuromuscul Disord. 2013;23(3):195-205

12. Graham RJ, Muntoni F, Hughes I, Yum SW, Kuntz NL, Yang ML et al. Mortality and respiratory support in X-linked myotubular myopathy: a RECENSUS retrospective analysis. Arch Dis Child. 2020;105(4):332-8.

13. Feingold B, Mahle WT, Auerbach S, Clemens P, Domenighetti AA, Jefferies JL, et al. Management of Cardiac Involvement Associated with Neuromuscular Diseases: a scientific statement from the American Heart Association. Circulation. 2017;136(13):e200-31.

14. Tayal U, Newsome S, Buchan R, Whiffin N, Halliday B, Lota A, et al. Phenotype and clinical outcomes of Titin cardiomyopathy. J Am Coll Cardiol. 2017;70(18):2264-74.

15. Cullup T, Lamont PJ, Cirak S, Damian MS, Wallefeld W, Gooding R, et al. Mutations in MYH7 cause multi-minicore disease (MmD) with variable cardiac involvement. Neuromuscul Disord. 2012;22(12):1096-104.

16. Finsterer J, Stollberger C, Brandau O, Laccone F, Bichler K, Laing NG. Novel MYH7 mutation associated with mild myopathy but life-threatening ventricular arrhythmias and noncompaction. Int J Cardiol. 2014;173(3):532-5.

17. Kiselev A, Vaz R, Knyazeva A, Khudiakov A, Tarnovskaya S, Liu J, et al. De novo mutations in FLNC leading to early-onset restrictive cardiomyopathy and congenital myopathy. Hum Mutat. 2018;39(9):1161-72.

18. Petri H, Wahbi K, Witting N, Kober L, Bundgaard H, Kamoun E, et al. Congenital myopathies are mainly associated with a mild cardiac phenotype. J Neurol. 2019;266(6):1367-75.

19. Ravenscroft G, Miyatake S, Lehtokari VL, Todd EJ, Vornanen P, Yau KS, et al. Mutations in KLHL40 are a frequent cause of severe autosomal-recessive nemaline myopathy. Am J Hum Genet. 2013;93(1):6-18.

20. Johnston JJ, Kelley RI, Crawford TO, Morton DH, Agarwala R, Koch T, et al. A novel nemaline myopathy in the Amish caused by a mutation in troponin T1. Am J Hum Genet. 2000;67(4):814-21.

21. Chang $X$, Jin $Y$, Zhao H, Huang Q, Wang J, Yuan Y, et al. Clinical features and ryanodine receptor type 1 gene mutation analysis in a Chinese family with central core disease. J Child Neurol. 2013;28(3):384-8.

22. Wang Q, Hu Z, Chang X, Yu M, Xie Z, Lv H, et al. Mutational and clinical spectrum in a cohort of Chinese patients with hereditary nemaline myopathy. Clin Genet. 2020.

23. Dubowitz V, Sewry CA, Oldfors A. Muscle biopsy: a Practicle approach. 4th ed. Edinburgh: Saunders/Elsevier; 2013.

24. Richards S, Aziz N, Bale S, Bick D, Das S, Gastier-Foster J, et al. Standards and guidelines for the interpretation of sequence variants: a joint consensus recommendation of the American College of Medical Genetics and Genomics and the Association for Molecular Pathology. Genet Med. 2015:17(5):405-24. 
25. Ryan MM, Schnell C, Strickland CD, Shield LK, Morgan G, lannaccone ST, et al. Nemaline myopathy: a clinical study of 143 cases. Ann Neurol. 2001;50(3):312-20.

26. McEntagart M, Parsons G, Buj-Bello A, Biancalana V, Fenton I, Little M, et al. Genotype-phenotype correlations in X-linked myotubular myopathy. Neuromuscul Disord. 2002;12(10):939-46.

27. Finsterer J, Stollberger C. Review of cardiac disease in Nemaline myopathy. Pediatr Neurol. 2015;53(6):473-7.

28. Marseglia L, D'Angelo G, Manti S, Salpietro V, Arrigo T, Cavallari V, et al. Sudden cardiac arrest in a child with nemaline myopathy. Ital J Pediatr. 2015:41:20.

29. Oates EC, Jones KJ, Donkervoort S, Charlton A, Brammah S, Smith JE 3rd, et al. Congenital Titinopathy: comprehensive characterization and pathogenic insights. Ann Neurol. 2018;83(6):1105-24.

30. Wang H, Castiglioni C, Kacar Bayram A, Fattori F, Pekuz S, Araneda D, et al. Insights from genotype-phenotype correlations by novel SPEG mutations causing centronuclear myopathy. Neuromuscul Disord. 2017;27(9):836-42.

31. Peddareddygari LR, Oberoi K, Sharer LR, Grewal RP. Adult diagnosis of type 1 Fiber predominance myopathy caused by novel mutations in the RYR1 gene. J Clin Neuromuscul Dis. 2019;20(4):214-6.

32. Samoes R, Oliveira J, Taipa R, Coelho T, Cardoso M, Goncalves A, et al. RYR1-related myopathies: clinical, Histopathologic and genetic heterogeneity among 17 patients from a Portuguese tertiary Centre. J Neuromuscul Dis. 2017;4(1):67-76.

33. Laughlin RS, Niu Z, Wieben E, Milone M. RYR1 causing distal myopathy. Mol Genet Genomic Med. 2017;5(6):800-4.

34. Loseth S, Voermans NC, Torbergsen T, Lillis S, Jonsrud C, Lindal S, et al. A novel late-onset axial myopathy associated with mutations in the skeletal muscle ryanodine receptor (RYR1) gene. J Neurol. 2013;260(6):1504-10.

35. Fox MD, Carson VJ, Feng HZ, Lawlor MW, Gray JT, Brigatti KW, et al. TNNT1 nemaline myopathy: natural history and therapeutic frontier. Hum Mol Genet. 2018;27(18):3272-82.

36. Géraud J, Dieterich K, Rendu J, Uro Coste E, Dobrzynski M, Marcorelle P, et al. Clinical phenotype and loss of the slow skeletal muscle troponin T in three new patients with recessive TNNT1 nemaline myopathy. J Med Genet. 2021;58(9):602-8.

\section{Publisher's Note}

Springer Nature remains neutral with regard to jurisdictional claims in published maps and institutional affiliations.

Ready to submit your research? Choose BMC and benefit from:

- fast, convenient online submission

- thorough peer review by experienced researchers in your field

- rapid publication on acceptance

- support for research data, including large and complex data types

- gold Open Access which fosters wider collaboration and increased citations

- maximum visibility for your research: over 100M website views per year

At BMC, research is always in progress.

Learn more biomedcentral.com/submissions 